\title{
Démythisation dialectique et reconstruction sémantique de la dyade maître-disciple dans L'Enfant-pluie de Francis Bebey
}

Éduquer un enfant, dit-on trivialement, c'est gagner un homme. Autrement dit, un enfant sans éducation, à défaut de devenir une menace sinon un danger pour les siens et pour son pays, traduit l'échec de la société en matière de préparation d'une relève susceptible de suppléer l'œuvre de développement amorcée par les aînés. On comprend donc pourquoi il s'établit entre l'enfant et son éducateur une interaction dialectique féconde qui suscite un questionnement heuristique. Comment s'opère le jeu didactique dans le cadre de la formation à laquelle souscrivent volontiers maitre et disciple en termes d'accompagnement et/ou de guidance ? Quels sont les enjeux interrelationnels entre disciple et maître dans la dynamique des échanges qui les lient?

L'horizon d'analyse à l'aune duquel nous négocions la réponse à cette problématique se réclame de la sociocritique d'Edmond Cros. Pour ce dernier, «le texte est un objet moral ; c'est l'écrit en tant qu'il participe au contrat social [... Il] émerge de la coïncidence conflictuelle de deux discours contradictoires [portant] l'un et l'autre sur des enjeux fondamentaux de la société : le génotexte et le phénotexte $"(2003$, p. 52).

Pierre Suzanne Eyenga Onana - maître de conférences à l'Université de Yaoundé I. Adresse de correspondance : Eyenga Onana Pierre Suzanne, S/C Père Rigobert Touye Binyegui, Paroisse de l’Omnisports, BP 185/C-451, Yaoundé, Cameroun ; e-mail : eyonapiers@gmail.com ORCID iD : https://orcid.org/0000-0002-2001-4472 


\section{Des considérations phénotextuelles et génotextuelles de l'apprentissage}

Considérés comme la strate énonciative grammaticalisée dans le dispositif sociocritique de Cros, les phénotextes "sont à envisager comme des formules de la signifiance dans la langue naturelle, comme des remaniements et des refontes successives du tissu de la langue " (Cros, 2003, p. 56). Commentant cette définition, Jacques Fame Ndongo perçoit le phénotexte comme le "texte audible et visible par tous " (2018, p. 55). Quant au génotexte, il décrit « l'espace virtuel où les structures originelles programment le processus de productivité sémiotique " (Cros, 2003, p. 55-56). Assimilable à la structure profonde, en linguistique il renvoie au « texte caché » (Fame Ndongo, 2018, p. 55). Dans l'analyse du texte de Francis Bebey, nous combinons phénotexte et génotexte pour donner plus d'effet à la signifiance. Le phénotexte est constitué par l'ensemble des stratégies qui définissent l'approche de Mwana dans sa quête de devenir adulte. Le génotexte évoque l'esthétique ou l'ensemble des moyens convoqués par le maitre tout comme le disciple pour jouer chacun sa partition avec efficacité.

\subsection{Des stratégies d'accès à la connaissance}

Pour réaliser son rêve cher de devenir grand, Mwana développe diverses stratégies allant du questionnement itératif à la scène en passant par le jeu de questions-réponses.

\subsubsection{La scène, une rhétorique du tâtonnement}

Généralement dialoguée dans la typologie de Genette, la scène tend à réaliser, sans l'incursion d'un personnage tiers, une égalité entre le temps de la narration et le temps de l'histoire, rendant les deux temporalités isochrones. C'est la scène qui réalise le gros de l'apprentissage du disciple dans sa quête de maturité auprès de sa grand-mère Iyo. Le disciple axe ledit apprentissage autour des questions multiples formulées sur la base d'une rhétorique du tâtonnement. Cette rhétorique consiste également pour le disciple à persister dans sa quête sapientiale en accablant son informateur de questions thématiques lui permettant de glaner des informations qui participent de sa formation holistique en vue de devenir un homme accompli. La clé du jeu repose sur tout un ensemble de questions appelant des réponses instantanées de la part du maître. À travers ce jeu inspiré du genre oral notamment du conte, le disciple apprend à quoi renvoie le concept de descendance qui, jusque-là, restait pour lui un mystère :

- Niko descend de l'Enfant-pluie.

- Il va tomber par terre?

- Non, Mwana. Quand je dis qu'il descend, cela signifie que c'est l'Enfant-pluie qui l'a engendré. 
- Niko ?

- Non ! L’Enfant-pluie était le grand-père de Niko [...] Tu sais, Niko n'est pas un des nôtres...

- D'où vient-il ?

- Lui est né ici...

- C’est ça le conte, Iyo, c'est ça?

- Tu as voulu que je te raconte l'histoire de l'Enfant-pluie....

- Oui, Iyo raconte.

- Le déluge, c'est quoi ? [...]

- Mais d’où vient ce déluge? [...]

- Tu as un grand-père, toi ? [...]

- Le bon Dieu, c'est la lune?»(Bebey, 1994, p. 29-30-34)

Il faut dire que la posture du maître échoit dans le texte à tout personnage capable de contribuer au moulage de la personnalité de Mwana. Car dans la formation de Mwana, il y a également l'apport de Yango ou son rapport à son cousin, mais aussi Ekalé, une patiente d'Iyo. Ces deux maîtres atypiques contribuent à leur manière à la formation sexuelle de Mwana par le biais de la scène. La figure de l'euphémisme leur permet de référer aux organes génitaux masculin et féminin respectivement à travers l'usage des lexèmes " commandant " et "palais ", afin d'éviter d'affecter la naïveté du disciple. L'art du dialogue doublé de la scène entre Mwana et Ekalé traduisent cette option pédagogique :

- Qui t’a dit que c'est comme ça qu'on fait des enfants?

- Yango. Il m’a tout enseigné.

- Enseigné comment ? [...]

- Il m’a appris que pour faire un enfant, il faut d'abord réveiller mon commandant. Quand le commandant est réveillé, il est debout. A ce moment-là, il faut que la reine lui ouvre son palais ;

- [...] En tout cas, ce n'est pas complètement faux. Regarde, voici le palais de la reine. Et toi, présente-moi ton commandant. (Bebey, 1994, p. 87)

L’apprentissage de Mwana se déroule par ailleurs dans le cadre de prospections.

\subsubsection{Les prospections}

La prolepse renvoie à « toute manœuvre narrative consistant à raconter ou évoquer d'avance un événement ultérieur» (Genette, 1972, p. 82). Le disciple s'en sert pour poser un contre-argument à celui d'un jeune maitre lorsque ce dernier le fait chanter. Tel est le cas pour Yango lorsqu'il surprend Ekalé et Mwana projetant l'acte sexuel. Alors qu'il menace de tout dévoiler, à travers une prolepse, Mwana lui donne la preuve de sa maturité en opposant une autre forme de chantage qui suscite l'admiration d'Ekalé qui voit désormais en lui un « homme » comme elle le lui déclare sur un ton 
métaphorico-emphatique empreint de comparaison : " tu parles comme un homme, Mwana! Tu es véritablement un homme maintenant " (Bebey, 1994, p. 89). Le disciple Mwana donne ainsi au maitre, Yango, à travers les verbes proleptiques « irai » et «fera ", la démonstration du niveau d'apprentissage, de maturité et de confiance où il se situe auprès d'Iyo lorsqu'il déclare à Ekalé : «j'irai lui parler [...] Je sais sur lui une toute petite chose qui le fera taire» (Bebey, 1994, p. 89). La prolepse n'est valide que lorsqu'elle se réalise dans la suite du récit. Mwana met effectivement sa menace à exécution obtenant en échange le silence de Yango, le maître-chanteur. Ce dernier, comme Ekalé, confirme que le disciple d'hier est devenu " grand ». À travers la prospection, Yango le lui affirme : «Bien, je vois que tu es devenu grand [...]. Et surtout, je ne raconterai pas ce que je vous ai vus faire, Ekalé et toi » (Bebey, 1994, p. 89). Autant arguer que le nouveau statut « d'homme » que revêt le disciple lui vaut désormais respect et considération. Car, pour parvenir au seuil de la grandeur et se faire (in)former, le disciple aura été exposé par son maître à des modes d'enseignement variés qu'il importe de scruter.

\subsection{Des modes d'enseignement}

Bloch et Wartburg dans Le Dictionnaire étymologique de la langue française, disent du participe présent " adolescere " qu'il signifie grandir. En effet, l'adolescent, tel Mwana, a " pour caractéristique essentielle dêtre en train de grandir " (Leif et Dalay, 1968, p. 17). Voilà pourquoi la pédagogie de l'inachèvement sied à son maître pour moduler sa formation. Elle rime moins à " apporter à lélève des connaissances que de former son jugement » (Leif et Dalay, 1968, p. 20). Autrement dit, l'inachèvement charrie la quête de découverte progressive chez l'adolescent puisque "l'ensemble des hommes [...], en face des adolescents, constituent la génération d'adultes » (Leif et Dalay, 1968, p. 18). Ainsi, l'« adultus ", traduit l'idée " qui marque que l'opération de grandir a atteint son terme" (Leif et Dalay, 1968, p. 17).

La démarche d'apprentissage établit un lien affectif entre maître et disciple, lequel se cristallise autour du projet que nourrit le disciple de devenir grand. Fasciné par son formateur, Mwana développe à son égard un sentiment d'admiration qui se voit par la richesse de son questionnement à son adresse. Il est d'ailleurs conscient qu'Iyo partage son phantasme de grandeur : « elle est au courant de la turbulence intérieure qui m'habite depuis que j'ai pris conscience de ma place au bas de l'échelle du savoir " (Bebey, 1994, p. 83). En retour, celle-ci le conditionne en suscitant l'auto-invention-de-soi-même consistant à amener le disciple à accoucher lui-même des réponses à ses préoccupations sapientiales. La dialectique maître-disciple revêt alors la forme de la maïeutique socratique dont les ressorts sont le refus de réponse à l'apprenant et la guidance.

\subsubsection{Le questionnement-guide}

L'accompagnement du maitre dans le cadre de l'apprentissage du disciple repose sur un questionnement formulé en sens inverse par le guide lorsque le disciple le soumet 
à un interrogatoire. Usant pour ainsi dire de l'ignorance socratique ${ }^{1}$ doublée d'ironie, Iyo feint de ne rien savoir. Au lieu de répondre directement à la question posée, elle entraîne Mwana dans une logique de pensée féconde mue par la stratégie de l'inférence. Il devra déduire la vérité qui git en lui-même en répondant à Iyo. À l'ouverture du récit, Iyo refuse de répondre au questionnement itératif de son disciple. Il s'agit de l'évocation en plusieurs temps d'un même énoncé interrogatif. Cette technique consiste pour le disciple à harceler son maître afin de lui arracher des réponses qu'il n'aurait pas autrement obtenues.

Cette forme de questionnement suscite quelques fois le dépit du maître. À la manière dont s'inaugure par une question le récit pathétique de Mina dans Sous la cendre le feu d'Evelyne Mpoudi Ngolle, ainsi le roman de Francis Bebey devient-il l'apanage du questionnement de type itératif à l'ouverture du récit. En formulant par trois fois la même question aux pages 5 et 7, Mwana opte pour la tactique du harcèlement. Elle vise à s'enquérir du fonctionnement des choses de la nature. Comme pour dire alors que « le langage est ce qui motive l'action, l'oriente et lui donne sens " (Charaudeau, 2005, p. 29), il s'enquiert auprès d'Iyo : "Est-ce que la pluie est vieille ? " (Bebey, 1994, p. 5). Lui-même, narrateur auto-homodiégétique, constate : «Iyo ne répond pas » (p. 5). Iyo sait pourtant que la pluie n'a pas d'âge mais suivant l'ironie qui définit la dialectique socratique, elle ne cède pas au harcèlement de son disciple. Au contraire, elle le guide dans son accouchement cognitif.

L'ironie consiste pour Iyo à manifester un léger courroux à l'adresse du disciple comme pour simuler une colère. L'usage de l'onomatopée et l'interrogation formulée à l'endroit de l'interlocuteur rejoignent les principes définitoires de la maïeutique socratique : «Aaaaaaaah! Yaaaaaaa ! [...] Quelle sorte d'enfant terrible es-tu donc, toi ! Tu vois bien que je suis occupée. Très occupée même; Et toi tu ne cesses de me poser des questions. Sans arrêt sans arrêt " (Bebey, 1994, p. 8). L'onomatopée "Aaaaah! Yaaaah! » qui introduit la réplique d'Iyo simule l'ennui voire la lassitude chez la maîtresse. Cela se voit aussi à l'usage itératif de l'expression "sans arrêt » et de la forme exclamative : "les enfants d'aujourd'hui posent de ces questions!" (Bebey, 1994, p. 9). Ces marques apparentes de vexation poussent pourtant le maitre à dispenser sa première leçon à un disciple avide de savoir. Iyo laisse l'apprenant entrevoir la possibilité d'une punition corrective au cas où la faute se reproduirait.

Dans L'Aventure ambiguë par exemple, le disciple Samba Diallo se voit violemment pincer le lobe de l'oreille par Thierno son maitre parce que, simplement, sa langue a fourché lors de la restitution d'un verset coranique. Pour un critique, cet épisode est la preuve qu' 'il n'y a aucune équivoque dans ce qu'il enseigne aux jeunes Diallobé à l'école coranique. Sa position somme toute bien tranchée, ignore toute autre alternative " (Meka, 2008, p. 51). Maître Thierno reste convaincu que la tolérance reste inopérante lors de la formation des jeunes aux choses sacrées car, affirme-t-il, « ce qu'ils apprennent vaut infiniment ce qu'ils oublient » (Kane, 1961, p.44).

1. Qui consiste à montrer que « tout ce que je sais c'est que je ne sais rien ». 
On peut ainsi convenir que les « principes d'altérité, d'influence et de régulation sont des principes fondateurs de l'acte de langage qui l'inscrivent dans un cadre actionnel dans une praxéologie d'agir sur l'autre " (Charadeau, 2005, p. 12). Le modèle pédagogique assumé par Iyo s'inscrit ainsi dans une double dynamique formative et informative. Elle déploie son enseignement sur un autre registre, à savoir le titillement et l'éveil de la conscience chez l'apprenant. Elle lui adresse en retour un questionnement, le poussant à en accoucher la vérité sur un fond de colère articulé sur une tonalité emphatique : "Mais qui t’a appris que les enfants doivent ainsi embêter leur grand-mère quand celle-ci travaille ? [...] Comment puis-je savoir l'âge de la pluie, moi » (Bebey, 1994, p. 8). Sans formellement indiquer au disciple comment se tenir en public notamment face à une personne occupée, Iyo joue la carte de l'intimidation visant à pousser le disciple à tirer les leçons qui s'imposent : " Le jour où tu reverras ton père, demande-lui donc si la pluie est vieille. Tu seras heureux de la réponse en coups de chicote qu'il te donnera. La pluie est vieille, la pluie est vieille » (Bebey, 1994, p. 8). La répétition du syntagme « la pluie est vieille » instaure une non-réponse apportée par le maître au disciple. La réponse se trouve contenue dans l'usage de l'interrogation rhétorique que formule Iyo : "Quelle femme irait mettre la pluie au monde ? Même très folle " (Bebey, 1994, p. 8). Jean-Jacques Robrieux affirme que ce type de question " n'appelle même pas de réponse, tant la réaction du public est considérée, même de manière forcée, comme évidente » (2000, p.116). Iyo n’espère donc aucune réponse de la part de Mwana. Elle sait en son for intérieur qu'il a non seulement appris comment se tenir prochainement en public mais aussi qu'il sait que la pluie n'a pas d'âge. En posant une dernière question rhétorique à son disciple, Iyo obtient enfin le résultat escompté. La réponse de l'apprenant Mwana conforte cet argument lorsqu'il constate lui-même que la pluie n'est pas tombée la veille. Dans la scène qui suit, l'usage de la conjonction donc dans l'intervention de Mwana, et de l'adverbe alors dans ceux d'Iyo, montre que l'apprentissage du disciple a porté des fruits :

- Quelle femme supporterait un enfant pareil ? Qui pleut qui pleut tout le temps.

- Pas tout le temps, Iyo ! Hier la pluie n'est pas tombée de toute la journée. Donc ${ }^{2}$ elle ne pleut pas tout le temps, n'est-ce pas vrai ?

- Alors, si elle n'est pas tombée hier, c'est qu'elle n'est pas vieille! (Bebey, 1994, p. 8)

L'autre mode d'enseignement que met à profit le maître est le dialogue socratique.

\subsubsection{Le dialogue socratique}

Marque de l'opposition constructive entre maître et disciple, le dialogue socratique traduit le soutient qu'apporte le maître au disciple dans sa quête. Selon le principe de l'intergénéricité qui avalise le mélange entre genres littéraires, et à travers l'usage du conte, Iyo expose Mwana à ses difficultés et l'aide simplement à se définir comme

2. Dans l'occurrence c'est moi qui souligne 
sujet potentiellement mature. Ses discussions avec lui revêtent une valeur éthique. Lorsqu'Iyo relate à son disciple l'histoire de l'Enfant-pluie, elle mentionne la nécessité d'invoquer le pardon du ciel et de la terre et de célébrer l'hospitalité, le disciple éprouve le besoin de comprendre le sens de ces expressions qui dépassent son entendement juvénile. Usant du temps du futur (comprendras, seras), Iyo renvoie son disciple à l'avenir dans leur dialogue :

- Qu'est-ce que cela veut dire?

- Mwana, c'est vrai, tu es encore trop petit. Tu comprendras ces choses-là plus tard, quand tu seras grand. (Bebey, 1994, p. 32)

La non-réponse du maitre instaure un mode de conduite nouveau chez le disciple dans sa recherche de la vérité. Il doit faire des efforts pour mériter le statut de grand afin d'accéder à certaines formes de savoir. Le défi qui naît en lui se voit à travers le soliloque ponctué du style direct afin de dévoiler les termes de son combat futur : " "Quand tu seras grand", répété-je dans mon for intérieur. Pourquoi y a-t-il tant de choses qui attendent que je sois grand! cela me rend furieux, mais je ne dis rien à ce sujet » (Bebey, 1994, p. 32). Cette fois-ci, le maître fait mystère de la réponse espérée du disciple. Il le renvoie carrément à une transcendance : « va donc demander tout ça au bon Dieu, Mwana. Lui seul pourrait te répondre " (Bebey, 1994, p. 34). Cette réponse vise à susciter une autre attitude de quête chez le disciple, celle de découvrir le logis de Dieu. Il s'agit pour le maître de faire fructifier un module de formation en initiant le disciple à une logique de quête permanente tant qu'une problématique reste non élucidée. Articulé sur une tonalité emphatique introduite par le présentatif c'est, le nouvel interrogatoire du disciple traduit cette quête :

- Il n'y a personne là-haut, dans le ciel.

- Même pas la lune?

- Le bon Dieu, c'est la lune? (Bebey, 1994, p. 34)

Narrateur auto-homodiégétique, le disciple découvre l'autre stratégie du maître consistant en l'alternance des silences et des réponses irrésolues, suggestives. La révélation du plan pédagogique du maître est faite à travers ses silences comme le souligne le disciple : "Iyo ne répond pas. Elle se contente seulement de me regarder " (Bebey, 1994, p. 34). Le silence du maitre est incitatif ; il invite le disciple à afficher un mode de comportement neuf auquel l'encourage d'ailleurs le maitre : «Toi, Mwana, tu grandiras avant la prochaine marée, [...] Pourvu qu' "ils" te laissent vivre en paix et longtemps » (Bebey, 1994, p. 34). Le décryptage par le disciple de la signifiance du mot « ils » articulant les propos du maitre autorise à proclamer le terme d'un patient processus de maturation. En se surpassant au plan cognitif, à travers le recours à un procédé d'inférence, le disciple parvient à percer les mystères qui entourent le « ils » afin de bien cerner le sens des propos du maitre. Ce décryptage illustre à quel 
point Mwana a grandi, autant en âge qu'en sagesse. À travers la reprise emphatique conduite par le présentatif " ce sont », le disciple clame sa grandeur puisqu'il peut désormais interpréter les choses du monde : «plus tard, quand je serai grand, j'apprendrai que "ils", ce sont les esprits» (Bebey, 1994, p. 34).

Parvenu à ce stade de grandeur, le disciple invite à interroger ses rapports à son maître dans le cadre de sa formation continue.

\section{Des rapports didactiques entre disciple et maître}

La didactique étudie " chacune des étapes de l'acte d'apprentissage et met en évidence l'importance du rôle de l'enseignant, comme médiateur entre l'élève et le savoir [...] C'est l'ensemble du processus construisant le rapport au savoir qui est analysé » (Vergnaud, 2001, p. 273). Le rapport didactique qui définit l'option pédagogique du maître permet d'établir la nature variable des rapports entre Mwana et Iyo. Ces rapports se fondent sur une dynamique ambivalente alternant déconstruction et construction.

\subsection{De la déconstruction du maître}

Dans un premier temps, on note un processus de déconstruction par le disciple du mythe que représente le maître à travers un affrontement inconscient fondé sur l'intensification d'un questionnement parfois diffus qui pousse le maître à un retranchement intellectuel afin de satisfaire les attentes de son disciple. Au questionnement du disciple, le maître en oppose un autre. Ainsi, à la question de savoir « qu'est-ce que c'est, "lire et écrire" " ? (Bebey, 1994, p. 15), Iyo, sans hésiter, pose à Mwana une autre question : " tu n'as encore jamais vu un livre ? [...] tu n'as jamais vu ton père lire la bible, cette chose noire avec une tranche rouge et des feuilles qu'on tourne comme ça, comme ça ?» (Bebey, 1994, p. 15).

Cette tactique évite au maître de se tromper dans sa tentative de répondre au disciple à propos d'un sujet dont les contours sémantiques lui échappent. Iyo définit l'acte d'écrire comme l'art « de mettre des histoires dans les livres. Et après quand tu as fini d'écrire, quelqu'un d'autre vient et prend ton livre et se met à lire ce que tu as écrit » (Bebey, 1994, p. 16). Cette définition du maittre trahit ses balbutiements face à la question embarrassante du disciple : « alors [...] les histoires que tu me racontes la nuit au moment où je vais m'endormir, tu peux les mettre dans la bible? ? (Bebey, 1994, p. 16). La preuve pour le disciple que le maitre est loin de toujours faire l'objet de sa fascination apparait lorsque ce dernier recourt au comique pour tenir la route dans le cadre d'un dialogue didactique. Le comique que convoque Iyo sur un arrière-fond d'emphase souligne la complexité pour le maitre de briller sur une problématique dont elle ne sait véritablement pas grand-chose : « cet enfant, cet enfant va nous tuer un jour avec ces questions qu'il pose à propos de tout, comme s'il était déjà grand. Éclat de rire sans réserve " (Bebey, 1994, p. 16). La réponse qui suit témoigne 
en outre de la gêne d'une femme analphabète qui doit néanmoins fournir une explication convaincante à un disciple débordant de curiosité : " mais moi que tu vois là devant toi, est-ce que je sais seulement écrire ? [...] Écrire, c'est l'affaire des gens qui vont à l'école et non des vieilles personnes comme moi. Toi tu sauras écrire, car un jour tu iras à lécole pour apprendre à mettre histoires dans les livres " (Bebey, 1994, p. 17). Lorsqu'on sait avec Charlot que «tout élève aborde un objet d'apprentissage à partir des relations qu'il peut établir avec cet objet d'apprentissage » (dans Jonnaert et Vander Borght, 2003, p. 49), on comprend pourquoi le maitre adopte une démarche courageuse visant à dévoiler son ignorance au disciple. Face à la persévérance de ce dernier, elle se veut rassurante et ironise en invoquant l'avenir à travers une prolepse : «Mwana, ta grand-mère ne sait pas lire. Ta vieille grand-mère ne sait pas écrire. Mais toi, Mwana, tu sauras lire et écrire ? Comme les Blancs venus de l'Ouest [...] Mais quand tu auras les yeux ouverts, Mwana, ne m'oublie pas » (Bebey, 1994, p. 17).

Malgré les carences cognitives du maître, ce dernier ne manque pas de travailler à la construction du disciple.

\subsection{De la construction du disciple}

Dans L'aventure ambiguë, le phénomène de construction réside pour Samba Diallo dans le fait d'aller apprendre, chez les Occidentaux, comment vaincre sans avoir raison. Mais il échoue, dévoilant la perte d'une identité qui s'avère intolérable pour le peuple des Diallobé : "il nous apparaît soudain que, tout au long de notre cheminement, nous n'avons pas cessé de nous métamorphoser, et, que nous voilà devenus autres" (Kane, 1961, p.125). Faute d'avoir pu se construire, la mort de Samba Diallo s'impose car il « est considéré comme un élément dangereux pour la survie de la communauté. [...] Sa mort s'impose donc comme une nécessité, pour sauver les apparences, à défaut de sauver Samba Diallo lui-même » (Meka, 2008, p. 49). Pour conjurer pareille déconvenue, Lapierre évoque le concept alternatif de référence fondamentale. Celui-ci souligne l'urgence pour les enseignants et les élèves de partager l'attitude essentielle dans la profession ou l'apprentissage d'une discipline concernée. Ce concept

représente la vision du monde, [...] ou regard intégré par l'enseignant lui-même et qu'il transmet à ses élèves implicitement ou explicitement. [...] L’enseignant a intégré cette attitude, il en est le modèle ; pour l'élève, il est le médiateur essentiel. Il fait entrer ses élèves dans cette profession ou cette discipline et lélève donne du sens à ce qu'il fait en saisissant cette attitude. (Lapierre, 2007, p. 41)

La vision du monde du romancier se révèle à travers l'attitude du maître. Ce dernier brûle d'envie de construire un homme singulier en projetant de façonner son disciple. Il s'agit de le mouler en un modèle exemplaire digne de suppléer les aînés. Cette vision consiste à guider le disciple à travers les méandres de la connaissance tout 
en ne le maintenant pas radicalement dans le mystère. Mwana résume parfaitement cette démarche qu'il perçoit difficilement et dont le point de chute est sa construction si l'on s'en tient au sens du mot « presque " qui nuance son affirmation dans ce soliloque :

Les grandes personnes savent beaucoup de choses mais n'expliquent presque rien aux enfants. Il faut grandir. Grandir jusqu'au jour où l'on sera capable de comprendre soimême. Tout comprendre tout seul. Ah, le jour où cela m'arrivera de tout comprendre tout seul, je montrerai à tout le monde que je suis enfin devenu grand. (Bebey, 1994, p. 55)

L'urgence de savoir et le désir de grandeur qui agitent la psychè du disciple l'entraînent dans une quête effrénée de connaissance dont le but final est de se voir considéré comme grand. Ceci explique pourquoi il interroge le maître sur tout. Sa hantise participe d'un défi qu'il entend relever à travers ces propos marqués d'emphase : « oui, j'aimerais être adulte tout de suite c'est vrai. Pour pouvoir dire aux petits enfants de mon âge comme Iyo ne cesse de me répéter : "vous comprendrez tout cela quand vous serez grands" »(Bebey, 1994, p. 55).

La construction du disciple intègre parfois certaines anti-valeurs telles que le mensonge positif. Ce vice consiste à couvrir l'erreur inconsciente d'un maître face à son disciple pour lui enseigner comment se préserver d'une punition assurée. Ayant inconsciemment volé des denrées au petit marché où l'avait envoyé sa tante Noëlla, Yango, jouant les maîtres de circonstance, évite la bastonnade et initie son disciple Mwana aux stratagèmes qui lui montrent les avantages du mensonge positif lorsque sa tante l'invite à la conduire audit marché : « il y a deux marchés, [...] le grand et le petit. Tanty Noëlla m'avait envoyé au petit marché [...] Moi je suis allé au grand pour faire les courses [...] où personne ne me reconnaîtrait » (Bebey, 1994, p. 132). La construction du disciple s'achève par le module d'apprentissage sur l'éthique comportementale. Celui-ci établit clairement que le mensonge, quel qu'il soit, constitue une enfreinte au vivre-ensemble et une violation de la loi stipulant que tout vol, conscient ou non, s'avère un acte repréhensible passible d'emprisonnement. Certes, le mensonge auquel a recours Yango, le maitre, lui évite la bastonnade mais il présente le désavantage d'être un contre-exemple qui nuit à la construction du disciple. Pour preuve, à la suite d'un autre vol de montre perpétré par Yango, le mauvais maître, celui-ci est arrêté et emprisonné. L'acte de construction du disciple se scelle par l'aveu de son inconduite qu'il formule à l'endroit du maitre. Complice de Yango lors du vol, Mwana démontre à Iyo qu'il a parachevé le processus de sa construction en se confondant en excuses nourries : « je ne ferai plus jamais cela, Iyo » (Bebey, 1994, p. 159).

Au demeurant, le rapport maitre-disciple se fonde sur des liens affectifs que le formateur nourrit face à l'apprenant. Pour construire la vie du disciple fasciné par la grande masse des savoirs du maître, ce dernier s'appuie sur les ressorts de la maïeutique socratique dont les stratégies le poussent à faire accoucher au dis- 
ciple la vérité qu'il recherche à travers un questionnement obsédant. L'accompagnement qui en résulte génère un homme accompli, rationnel, capable de trouver des solutions aux problèmes qu'il soulève. En reconnaissant sa dernière erreur face à son maître de toujours, Mwana prouve sa maturité et montre que le principe d'isomorphisme se vérifie, d'après lequel « les savoirs à l'œuvre dans l'action didactique doivent être organisés selon les mêmes lignes de force ; leur logique interne, c'est-àdire la problématique des valeurs et des finalités qui les déterminera, sera la même » (Raisky, 1998, p. 54).

\section{RÉFÉRENCES}

Bebey, F. (1994). L'Enfant-pluie. Yaoundé : CLÉ.

Bloch, O., Walther, W. (2008). Dictionnaire étymologique de la langue française. Paris : PUF. Charaudeau, P. (2005). Le Discours politique. Les masques du pouvoir. Paris : Vuibert.

Cros, E. (2003). La Sociocritique. Paris : L'Harmattan.

Fame Ndongo, J. (2018). Le Phénomène Paul Biya. Essai de sémiotique arithmétique. Yaoundé: Editafric.

Genette, G. (1972). Figures III. Paris : Seuil.

Hugo, V. (1966). Les Orientales. Paris : Gallimard.

Jonnaert, P., Vander Borght, C. (2003). Créer des conditions d'apprentissage : un cadre de référence socioconstructiviste pour une formation didactique des enseignants. Bruxelles : De Boeck Université.

Kane Hamidou, C. (1961). L'Aventure ambiguë. Paris : Présence Africaine.

Lapierre, L. (2007). Guide d'accompagnement du cours DID 868 à l'intention des répondantes et des répondants locaux de PERFORMA. Sherbrooke : Cégep de Sherbrooke et Performa.

Leif, J., Delay, J. (1868). Psychologie et éducation. L'adolescent. Paris : Fernand Nathan.

Meka Obam, J.-M. (2008). La Structure symbolique dans L'aventure ambiguë de Cheikh Hamidou Kane et Le monde s'effondre de Chinua Achebe. Paris : L'Harmattan.

Raisky, C. (2001). Doit-on en finir avec la transposition didactique ? Dans J.-C. RuanoBorbalan (dir.), Éduquer et former (p. 50-64). Paris : Éditions Sciences humaines.

Robrieux, J.-J. (2000). Rhétorique et argumentation. Paris : Nathan.

Vergnaud, G. (2001). Piaget visité par la didactique. Intellectica, 33, 107-123.

RÉSUMÉ : Dans L'Enfant-pluie de Francis Bebey, l'apprenant Mwana brûle d'envie de devenir grand. Sevré pour ainsi dire de ses parents, il est obligé de vivre au village aux côtés de sa grand-mère Iyo. Il fonde sa quête sapientiale journalière sur un questionnement répétitif qu'il formule à l'endroit de son maître. Mais par-delà la fascination de l'enfant et face à l'immensité des modules de formation, quels types de liens singularisent-ils son rapport à Iyo ? Un accompagnement didactique pour le maitre ou simplement une guidance pour l'apprenant ? En adossant notre analyse au diptyque phénotexte-génotexte qui sous-tend l'approche sociocritique d'Edmond 
Cros, nous organisons notre travail en deux grandes parties. D'abord, nous scrutons les composantes thématiques qui articulent l'apprentissage de Mwana en montrant à chaque fois comment l'artiste-écrivain stylise cette thématique pour enfin interroger la vision du monde qu'il postule à travers son écriture.

Mots-clés : démythisation, accompagnement didactique, dialectique, sociocritique, vision du monde, Francis Bebey

\section{Dialectical demystification and semantic reconstruction of the master-disciple dyad in Francis Bebey's L'Enfant-pluie}

ABSTRACT: In Francis Bebey's L'Enfant-pluie, the learner Mwana is burning with the desire to grow up. Weaned almost from his parents, he is forced to live in the village with his grandmother Iyo. He bases his daily sapiential quest on a repetitive questioning that he formulates towards his master. But beyond the child's fascination and in the face of the immensity of the training modules, what types of links do they singularize their relationship with Iyo? A didactic support for the teacher or simply a guidance for the learner? By backing our analysis with the phenotext-genotext diptych that underlies Edmond Cros' sociocritical approach, we organize our work into two main parts. First, we examine the thematic components that articulate Mwana's learning by showing each time how the artist-writer stylizes this theme to finally question the vision of the world he posits through his writing.

Keywords: demythisation, didactic support, dialectic, sociocritical, world view, Francis Bebey 